\title{
Improvements in the Method of Radiation Anomaly Detection by Spectral Comparison Ratios
}

D. M. Pfund ${ }^{*}$, , K.K. Anderson ${ }^{\mathrm{a}}$, R.S. Detwiler ${ }^{\mathrm{a}}$, K.D. Jarman ${ }^{\mathrm{a}}$, B.S. McDonald ${ }^{\mathrm{a}}$, B.D. Milbrath ${ }^{\mathrm{a}}$, M.J.

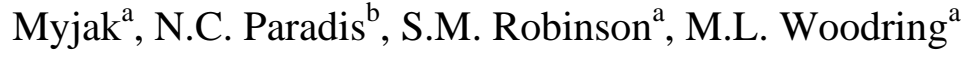

${ }^{a}$ Pacific Northwest National Laboratory, P.O. Box 999, Richland, WA 99352 USA

${ }^{b}$ U.S. Navy Space and Naval Warfare Systems Command, 53560 Hull Street, San Diego, CA 92152 USA *Corresponding author: Tel. No: 509-375-3879, e-mail: david.pfund@pnnl.gov

\begin{abstract}
We present a new procedure for configuring the Nuisance-rejection Spectral Comparison Ratio Anomaly Detection (N-SCRAD) method. The procedure minimizes detectable count rates of source spectra at a specified false positive rate using simulated annealing. We also present a new method for correcting the estimates of background variability used in N-SCRAD to current conditions of the total count rate. The correction lowers detection thresholds for a specified false positive rate, enabling greater sensitivity to targets.
\end{abstract}

Keywords: Anomaly detection, Gamma-ray spectroscopy, Radiation monitoring. 


\section{Introduction}

Gamma-ray spectroscopy has applications in many national security missions (Fagan et al. 2012), including imaging or localizing nuclear materials, and identifying constituent isotopes. Here we focus on the problem of detection during search operations, rather than - or as a precursor to - location or identification of illicit radiation sources (Runkle et al. 2009a). The problem of detecting threat signatures is compounded by difficulties, including those of estimating background radiation and supplying useful information to search operators. In most cases, the detection algorithm must be tailored before use to maximize its power to detect items of interest in a few broadly defined classes of radiation sources while reducing or constraining the rate of false positives.

The detection problem is one of separating expected and unexpected samples of, or contributions to, observed spectra. Aage et al. discussed the application of noise adjusted singular value decomposition (NASVD) to the detection and mapping of low levels of ${ }^{137} \mathrm{Cs}$ contamination from the Chernobyl accident. Their approach was to use a linear combination of principal components to estimate the contributions to observations from abnormal ${ }^{137} \mathrm{Cs}$ sources and from ordinary statistical noise, ${ }^{40} \mathrm{~K}$, U-series, Th-series sources. They applied a similar approach to the analysis of data collected by a car-borne $\mathrm{NaI}(\mathrm{Tl})$ detector, together with a visualization method to help operators identify targets (Aage and Korsbech, 2003). Guillot developed a filtering method of analyzing spectrum profiles for peaks. Energy windows were then examined for the presence of radioisotopes - both naturally occurring and presumed manmade. Hjerpe and Samuelsson examined vehicle-borne data for spectral indications of, and distances to, target ${ }^{137} \mathrm{Cs}$ sources. They used a energy windowing method that corrected counts observed in a ${ }^{137} \mathrm{Cs}$ window for background counts observed in a ${ }^{40} \mathrm{~K}$ window, and applied a curve fit to the temporal profile of collected counts to estimate distance to the target. None of these early approaches considered the problem of estimating the confidence level or statistical significance to assign to a presumed spectral abnormality, or how to decide if an alarm or alert level had been crossed in a search operation. 
From 2006 through 2010 we developed what we now call "Nuisance rejection spectral comparison ratio anomaly detection" (N-SCRAD) for flagging non-background gamma ray spectra collected from mediumresolution detectors during searches for illicit nuclear materials (Pfund et al. 2007; Anderson et al. 2008; Jarman et al. 2008; Pfund et al. 2010; Runkle et al. 2009b). The algorithm was developed for use in mobile searches, with detectors either mounted in vehicles or carried by people on foot. N-SCRAD combines exponentially weighted moving average (EWMA) tracking of expected background with a shape-based comparison of observations to the expected background to identify anomalies. Because the ability to resolve peaks from threat contributions is limited, we reduced spectra to counts collected in a few broad spectral regions of interest (ROIs). In N-SCRAD we do not apply a model for the spectral signatures of threats. However, we do choose ROIs in an attempt to maximize the sensitivity of the algorithm to prospective threats while minimizing sensitivity to benign radiation. The anomaly metrics (called alarm test statistics in this work) developed from count rates in ROIs conformed fairly well to a known chi-distribution, if the data were from background sources, and so the significance level of an observation yielding a large metric was also fairly well known as discussed below. In the intervening years we have continued to refine the algorithm, as we applied it to practical problems. The purpose of this paper is to describe those improvements.

Recently, other approaches to detecting anomalous spectral radiological signatures in low-count search and unattended monitoring scenarios have been studied. Reinhart incorporated spectral comparison ratios into a spatial anomaly mapping system (SCRAM) based on mobile detectors spread over a wide area, and modified the estimation of expected variation to allow for very limited prior measurements. Chan et al. presented a nonparametric approach that allows for deviations of background counts from assumed Poisson distributions, using the concept of a Brownian Bridge. Research continued to advance in fundamental areas, including assessing the information content of spectra as a function of detector resolution (Nelson et al. 2010; Jordan et al. 2011) and displaying of information to search operators (Kock et al. 2011). Yet improvements still need to be made in the fundamental areas of detection alarm test significance and power, particularly in applications with highly variable radiation backgrounds. 
Two particular problems are addressed in this paper - the first, constructing sensitive tests in the face of possible uncertainty in the threat signatures; the second, reducing false positives in the face of unanticipated, sudden changes in the radiation background. With regard to the first problem, in some cases the space of threats may include only a few items with known spectral signatures. In other cases, search operators may have much less knowledge of likely spectra from threat objects. Unanticipated sudden background changes can have operational consequences. Gradual changes can be tracked with a temporal filter, such as EWMA. Sudden changes in the shape of background spectra can often be ignored provided they are caused by changes in the relative abundance of naturally occurring radioactive materials (NORM). Unanticipated large changes in background total count rate, when coupled with even minor changes in spectrum shape or noise can trigger false alarms. In a search operation such alarms can be a significant diversion, as will be illustrated in Section 6 below.

High values of the N-SCRAD test statistics generate alarm or alert indications for search operators. Each such test statistic was a covariance-weighted length of a vector of spectral comparison ratios (SCRs), with each ratio being determined from counts collected in a pair of spectral regions of interest. The placements of the regions were engineered to collect counts from potential targets that were significantly different than the background variability likely to be observed in a search operation. We presented one method for optimizing such placements in our early work and illustrated its application assuming targets represented by ${ }^{133} \mathrm{Ba}$ and depleted uranium (DU) spectra (Pfund et al. 2007). However, in a typical application we may have many more than two representative threat surrogates, and it is likely that a single test statistic is inadequate for detecting all of them. In Section 2 of this work we present our latest, improved method for determining regions of interest and related detection algorithm parameters. The problem of determining parameters in detection algorithms in a general way was addressed by Portnoy et al. who applied a genetic algorithm. They were primarily interested in finding optimal alarm thresholds or alert levels based on measured benign source data. Our task here is one of clustering of threat spectra into groups and assigning of ROIs to the groups, for which we apply simulating annealing. Our objective function is based on estimates of minimal detectable count rates. 
Key to the good performance of N-SCRAD was the development of alarm test statistics such that those resulting from benign or background spectra appeared with frequencies described by chi-distributions (Pfund et al. 2007; Pfund et al. 2010). One step towards that goal was the elimination of long-tail, nuisance source events that can trigger false positive alarms. The most frequent of such events are triggered by the passage of naturally occurring radioactive material (NORM). In N-SCRAD, indicated anomalies in observed spectra caused by changes in NORM abundance are screened out. The algorithm can be configured to filter out other nuisance source signatures - such as those from medical isotopes or industrial sources - as needed for the search application.

Another step in obtaining predictable test statistics for benign sources is their proper standardization. Originally, N-SCRAD test statistics presented to operators during a search were standardized by covariance estimates made from previously collected background spectra with an EWMA routine. We have recently found that estimates made from past background conditions were not always good predictors of the covariance at current search conditions. Large, unanticipated changes in background radiation level often resulted in false positive detections using N-SCRAD, triggered by jumps in the alarm test statistics at background changes. In Section 5 of this work we present a correction to the covariance estimated by EWMA that improves the performance of the detection algorithm.

\section{N-SCRAD Optimization by Simulated Annealing}

In N-SCRAD we used several test statistics to trigger alarm or action events. Each test was tailored to particular classes of radionuclides. In our earlier work we envisioned HEU-like, DU-like and Pu-like classes of special nuclear materials (SNM) (Pfund et al. 2010). We assigned threat surrogate materials to these classes, or "threat clusters" manually and used an optimization routine to determine spectral regions of interest (ROIs) for each cluster. Areas for improvement included optimizing the number of test statistics and ROIs, developing a formal notion of spectral similarity to automate the clustering of threat spectra, and including a broad range of radiological threat spectra, SNM spectra, with shielding variations, in the clustering scheme. 
In our first paper, we used twelve ROIs for the detection of threats similar to two (DU and ${ }^{133} \mathrm{Ba}$ ) surrogates (Pfund et al. 2007). We introduced the idea of threat clustering, applying three test statistics with six to eight ROIs assigned to each (Pfund et al. 2010). However, because of variations in spectral shape with shielding, each of three clusters contained species with dissimilar appearing spectra. The three SNM-like clusters had no clear place for other potential target radionuclides such as ${ }^{137} \mathrm{Cs}$ and ${ }^{60} \mathrm{Co}$. We needed a new approach for parameterizing N-SCRAD that would automatically cluster numerous threat surrogates, create the associated test statistics, and determine the numbers and locations of spectral ROIs.

For this purpose we have adopted a parameter optimization by simulated annealing (SA) (Kirkpatrick et al. 1983; Henderson et al. 2003; Suman and Kumar, 2006). This method replaced the Powell's method of continuous variable optimization that we used in our earlier work for locating ROIs (Press et al. 2002). Unlike Powell's algorithm, SA can optimize over discrete variables and can explore over wide areas of the parameter space in a single run.

The SA software minimized an objective function $L$ that was a sum of estimated minimum detectable count (MDC) rates for all of the surrogate threats in our design basis for parameter selection, giving each equal weight:

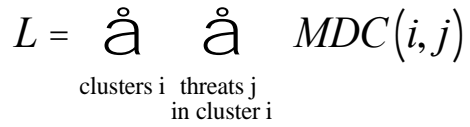

Here, each $M D C(i, j)$ was a theoretical minimum detectable count rate for surrogate $j$ in cluster $i$ at $95 \%$ probability of detection (PD) and $0.05 \%$ nuisance and false alarm probability (NFAP). The false positive specification prevented an overabundance of test statistics and ROIs. The above summations counted each surrogate only once - for the cluster that resulted in the lowest MDC value. The theoretical MDCs were given by,

$$
\operatorname{MDC}(i, j)=(1000 \text { counts }) \sqrt{\frac{\left(n_{i}\right)}{F O M(i, j)}}
$$


where $n_{\mathrm{i}}$ is the number of ROIs in cluster $i, \theta\left(n_{i}\right)$ is the value of the non-centrality parameter of a non-central chisquare distribution with $\left(n_{i}-1\right.$ - number of nuisances signatures rejected by $N$-SCRAD) degrees of freedom that places $95 \%$ of the distribution beyond the alarm threshold. The alarm threshold in this case was the value that contains [1-(NFAP/number of clusters)] fraction of a (central) chi-square distribution with the same number of degrees of freedom. The figure of merit (FOM) was defined as in our earlier work (Pfund et al. 2007),

$$
F O M(i, j)=\mathbf{a}_{j}^{\mathrm{T}} \cdot \Sigma_{i}^{-1} \bullet\left[\left(\mathbf{I}-\mathbf{P}_{i}\right) \cdot \mathbf{a}_{j}\right]
$$

where $\square_{\mathrm{j}}$ is the spectral comparison ratio (SCR) vector for the surrogate spectrum $j$ in cluster $i$ evaluated at 1000 total counts of the surrogate and $\mathbf{P}_{\mathrm{i}}$ is the nuisance source rejection transformation. The development of threat source templates $\mathbf{a}_{\mathrm{j}}$ for use in the optimization is discussed in Section IV below. The covariance $\boldsymbol{\Sigma}_{\mathrm{i}}$ was estimated from measured background spectra, the collection of which is also discussed in Section IV.

The theoretical MDC estimates in (1) were based on assumptions that SCR vectors in the background population are distributed approximately multivariate normal, that addition of counts from targets has little effect on the covariance matrix $\Sigma_{\square}$, and that the cluster test statistics for background spectra are independent (Pfund et al. 2007). The assumption of normality results in N-SCRAD test statistics for background samples being distributed chi-square, while those for a particular low-count rate target superimposed on background would be approximately distributed non-central chi-square. The assumption of normality here ignores the long tails in the distributions of the SCRs - tails that are enlarged by encounters with unexpected and uncharacterized nuisance sources.

At the input stage of the SA optimization, the input background data were reduced to two statistics - the background average and background variance in channels of the spectra. The template SCRs for the targets $\mathbf{a}_{j}$ and the background covariance matrix $\Sigma$ for each trial in the optimization were calculated from the two aggregate statistics that broadly characterize background, for use in (3). The non-centrality parameters $\theta$ in (2) were also calculated from the aggregate statistics. Any representative subset of the available data - including the whole set - 
can be used in the optimization without fear of over-training, as the parameter determination uses only the two aggregate statistics together with the assumption that the SCRs are normally distributed.

The SA algorithm minimized the objective function $L$ by making discrete parameter changes or "moves." In our case three types of moves in parameter values were possible: 1) A single-channel shift of either the left or right boundary of an ROI; 2) An ROI in a cluster is turned off (an SCR is no longer calculated from it) or turned back on; 3) A cluster is turned off (and not used to calculate any theoretical MDC values) or turned back on. After a move, theoretical MDC values were calculated for every surrogate in every active cluster and the objective function $L$ was determined. Our implementation of SA kept a running record of the parameter set that gave the lowest value of $L$ during execution, and output that set when the SA run was complete.

The SA program also accepted some parameter moves that increased the objective function, giving the algorithm some possibility of broadening its search or escaping local minima. The probability of accepting a briefly unfavorable move decreased with the resulting increase in the objective function and increased with the value of an internal "temperature" variable $T$ (Kirkpatrick et al. 1983). In this work, the initial temperature was set to the objective function value resulting from the initial guesses of ROI and threat clustering parameters. As the optimization continued the temperature was gradually lowered, eventually freezing the parameters at final values. A string of non-acceptances suggested the program converged to a local minimum and needed to look elsewhere in the parameter space for a better result. When a string of 200 or more such failed trials was detected, the program doubled the temperature and continued running, an operation referred to in the SA literature as reheating or reannealing (Anagnostopoulos et al. 2006). The variables that control the SA optimization program are listed in Table 1.

\section{Data For N-SCRAD Optimization}

Measured background data for N-SCRAD parameter optimization consisted of a set of 41,286 spectra collected in the San Diego area in May and June of 2013. Dynamic backgrounds were measured in the greater San Diego area, with the detector riding in the bed of a pickup truck. The truck drove through San Diego, La 
Jolla, Coronado Island and rural Wildcat Canyon Road areas. The detector system used was designed by the United States Navy Space and Naval Warfare Systems Command (SPAWAR), and was based on a 50mm x 100mm x 410mm (2"x4"x16") sodium iodide (NaI(Tl)) crystal. The detector acquired histogrammed spectra in 1024 channels at a rate of one spectrum per second, synchronized with time stamps and detector latitudes and longitudes from a global positioning system (GPS) unit. The detector employed an automatic calibration algorithm using a course-gain temperature feedback loop and a fine-gain ${ }^{40} \mathrm{~K}$ peak-tracking loop. The initial calibration curve of the detector was determined with the aid of long-dwell spectra from ${ }^{241} \mathrm{Am},{ }^{133} \mathrm{Ba},{ }^{137} \mathrm{Cs},{ }^{232} \mathrm{Th}$ test sources, used together with the background ${ }^{40} \mathrm{~K}$ peak. The long-dwell source measurements were done with the detector mounted in the pickup - as for the background measurements - as part of the June, 2013 measurement campaign.

The measured backgrounds used in estimating the covariance in (3) during parameter optimization should have variability that is representative of what is expected during a search application. The San Diego background data included spectra from urban, rural, and light industrial areas taken from a variety of road types from highway to dirt. The combined set contained at least three instances of likely anomalies caused by nuisance sources, two of which were identified as ${ }^{99 \mathrm{~m}} \mathrm{Tc}$ with PeakEasy version 4.40 spectral analysis software (Los Alamos National Laboratory). The data also had relevant background count rate variability associated with obvious geographic features and man-made structures. We matched the GPS locations with spectra and associated the jumps in background with passage over the Coronado Bridge, transit of Shelter Island (probably built with at least two types of fill material), driving under concrete overpasses, driving through gaps in Wildcat Canyon and passing down a street in downtown San Diego between tall brick-faced buildings. The total (gross) count rate in 1-second integrations versus spectrum index in the combined set of 41,286 background spectra is plotted in Figure 1. The two ${ }^{99 \mathrm{~m}} \mathrm{Tc}$ signatures were in spectra numbers 5703 and 20,202. The third nuisance signature (possibly ${ }^{137} \mathrm{Cs}$ ) signature was in spectrum number 24,778 .

The parameter optimization also used simulated spectra from surrogate threat sources, from which the templates $\mathbf{a}_{\mathrm{j}}$ used in (3) were developed. For this project, spectra from surrogate threat source materials as would 
be seen on a search vehicle were simulated with the Monte Carlo N-Particle photon transport code, MCNP version 5 (MCNP X-5 Monte Carlo Analysis Team). These spectra were used in selecting N-SCRAD test statistics and spectral ROI by simulated annealing, as discussed above. They were also used in quantifying the sensitivity of the detection algorithm to threat source counts injected into the measured background data. Our MCNP model included detector and search vehicle components. The detector model was based on engineering drawings of the unit furnished by SPAWAR. The model pickup truck was based on specifications for a Ford F350 and included frame and body mounts, bumpers, engine and power train, radiator and coolant, suspension, axles, brakes, wheels and tires, body, firewall, doors, windows, and fuel. This model vehicle sat in a simulated environment of air on a large non-emitting pad of pavement. As in the real system tested, the virtual detector sat in the bed of the pickup in the MCNP model. The truck body partially shielded the detector from the external surrogate sources, reducing the observed count rate and altering the shape of simulated spectra. Each model threat surrogate source was placed 9 meters (30 feet) from the center of the long face of the detector and 0.8 meters ( 2.65 feet) off the ground, floating in the air as a point source.

Our MCNP model was used to produce simulated spectra for 28 SNM and radiological threat surrogate sources that we believe have signatures that are representative or span those of real threats. The set included depleted uranium, highly enriched uranium (HEU), virgin highly enriched uranium, reactor and weapons grade plutonium, all with various shielding. The set also included single isotope bare ${ }^{241} \mathrm{Am},{ }^{60} \mathrm{Co},{ }^{137} \mathrm{Cs},{ }^{192} \mathrm{Ir}$ and ${ }^{133} \mathrm{Ba}$ sources. The purpose of using 28 sources was to broadly define what we mean by an anomalous spectrum when selecting test statistics and ROIs with the SA algorithm

In addition to the threat surrogates, spectra from NORM sources were simulated, for use in calculating the nuisance projections $\mathbf{P}_{\mathrm{i}}$ in (3) during optimization. The earth-shielded background components from ${ }^{40} \mathrm{~K}$ and the $\mathrm{U}$ and Th decay chains were simulated in MCNP, within models of soil deep enough that self-shielding effects rather than additional depth of the model limit further emissions. These NORM spectra were modeled beginning with the emission lines from all decay chains and then shielding these emissions with several meters of earth. Modeled potassium (K), uranium (U), and thorium (Th) spectra were ultimately used in computing N-SCRAD 
nuisance rejection transformations. During N-SCRAD operation, rapid changes in environmental spectra caused by changes in the local abundance of $\mathrm{K}, \mathrm{U}$ and Th are projected out before alarm test statistics are calculated, as a means of reducing the false positive rate, as previously documented.

\section{Optimization Algorithm Behavior}

The SA algorithm converged rapidly with best parameter estimates stabilized in less than 1000 cooling steps. Plotted in the upper half of Figure 2, on the primary axis, is the SA temperature versus step number. The sawtooth temperature increases indicate reheating that the program initiated to prevent trapping into a local minimum. Reheating appears to improve the convergence of SA in this application, by forcing the program to continue exploring the parameter space. The first reheating operations occurred after step 200, resulting additional small reductions in the objective function. The objective function versus step number is plotted on the secondary axis. The grey line is the running value of the objective; the black line is the best of prior steps. In the case shown, reheating produced an additional small reduction in the objective function around step 604. Another small reduction in MDC occurred at time step 765, resulting in the final parameter estimates. Small reductions in the objective function can be the result of large reductions in MDCs for particular surrogates. Plotted in the lower half of Figure 2 are the MDC estimates from (2) for three of the surrogates versus step number. Parameter changes after the reheating near step 604 lowered the MDC for surrogate A by $11 \%$ - MDCs for surrogates B and C did not decrease at that time.

The SA program performed dimensional reduction by discarding and or replacing ineffective trial test statistics and ROIs. Early in the parameter optimization, the SA distributed the 28 surrogates among the maximum number of ten clusters, with an associated large number of ROIs (at times over 110). Within 300 temperature steps most of these trial parameters were discarded, leaving 3 threat clusters (and associated alarm test statistics) with 25 to 30 ROIs between them. Plotted in grey in the upper half of Figure 3 is number of clusters and associated test statistics versus temperature step; the black line is the best value from prior steps. The three large reheatings near the end of the run activated more than three clusters, but the SA returned each 
time to the final value of three. Plotted in grey in the lower half of the figure is the total number of ROIs in use versus step number. The black line is the number of ROIs associated with the lowest MDC from prior steps. Near step 604 the program added two additional ROIs (going from 27 to 29). This change was correlated with the slight reduction in the objective function at that time, and so were accepted. The final number of ROIs was associated with the lowest MDC at time step 765. The SA program did not accept trial reductions in the number of ROIs at times 915 and 920 as they were associated with increases in the MDC.

The MDC estimates produced at the end of the optimization were lower than those obtainable with a naïve choice for the regions of interest and test statistic. We compared MDC estimates for the 28 surrogates to those similarly estimated using 35 ROIs with widths that followed a square root energy relationship. The simple unoptimized ROIs were constructed as discussed elsewhere, with one ROI centered on $662 \mathrm{keV}$ and the others spaced assuming a 9 percent energy resolution (Runkle et al. 2009c). We assumed that a single N-SCRAD test statistic would be used with the simple ROIs, with no clustering of threats by type. Black bars in Figure 4 are the estimated MDC values for the surrogates, in counts per second, that would be obtained from N-SCRAD using the naive choice of ROIs. Grey bars are the MDCs estimated with the optimized ROIs divided into the three optimized threat clusters. Estimated MDCs for the optimized N-SCRAD average 18.6\% lower than those for the naïve choice. The average improvement with optimization was $19.5 \%$ for surrogates with MDCs of greater that $400 \mathrm{cps}$, and $17.5 \%$ for surrogates with MDCs less that $400 \mathrm{cps}$.

As the ROI locations were optimized, the SA software performed feature selection on threat surrogate spectra, nuisance spectra, and background spectra, with the aim of enhancing sensitivity to the wide range of potential targets. It is likely that the N-SCRAD detection algorithm operating with parameters so broadly optimized will not be as sensitive as it would be when the presumed target space is smaller. However, detection performance when optimized for a broad range of surrogates will be close to what is obtainable with a more narrowly focused algorithm, one requiring more prior information about the search target. Black bars in Figure 5 are MDC estimates from (2) for two surrogates, based on N-SCRAD parameters developed from all 28 surrogate spectra in the algorithm design basis. Dark grey bars are MDC estimates obtained from subsets of the 28 that were 
isotopically similar to surrogate $\mathrm{D}$ on the left, and surrogate $\mathrm{E}$ on the right. The restricted sets still cover a wide range of source shielding. The reduction in MDC obtained by narrowing the space of threats to specific isotopes was $2 \%$ for surrogate $\mathrm{D}$ and $8 \%$ for surrogate $\mathrm{E}$. These reductions quantify the value in terms of sensitivity in knowing the nature of the material being searched for. The light grey bars are the MDC estimates obtained with detection parameters optimized on surrogate D only (on the left) or surrogate E only on the right, each with its own known shielding. These values are $13 \%$ less and $27 \%$ less, respectively than the MDCs obtained with general parameters. Some significant gains in sensitivity can be realized if searchers know both the radionuclides in, and the shielding on, the target. However, we believe that in most scenarios the shielding will be unknown. Assuming that is the case, there is little advantage to narrowing the space of threats to particular isotopes when optimizing N-SCRAD.

\section{Improved Covariance Estimate}

Each of the N-SCRAD test statistics at time index $k$, had the following form in our previous work,

$$
d_{i_{k}}^{2}=\boldsymbol{\alpha}_{i_{k}}^{\mathrm{T}} \cdot\left(\Sigma_{i_{k}}^{\text {tracking }}\right)^{-1} \cdot\left(\mathbf{I}-\mathbf{P}_{i_{k}}\right) \cdot \boldsymbol{\alpha}_{i_{k}}
$$

where $\boldsymbol{\alpha}_{\mathrm{i}}$ is a vector of spectral comparison ratios (Pfund et al. 2007;Anderson et al. 2008; Ely et al. 2006) and $\Sigma^{\text {tracking }}$ is an estimated background covariance matrix. (I-P-P $)$ is the nuisance rejection transformation. In our earlier work, the covariance matrix was estimated from spectra observed at previous time steps $k-1, k-2, \ldots$ with an exponentially weighted moving average (EWMA) background tracking routine. Background covariance estimates based on the recent past can have large systematic errors that bias the test statistics in (4) and so generate false positive indications. We observed in applications that as operators approached high count rate benign objects (granite blocks for example) the test statistics from (4) would increase rapidly together with the rapid increase in total count rate. The nuisance rejection procedure did not prevent the resulting anomalous indications. The estimated covariance matrix, based on past times, did not increase with increasing background total count rate as it should have. 
To eliminate such false positives we developed a method to correct background covariance estimates from past measurements to current conditions. We still consider that the recent past can be used to estimate the shape of the mean background spectrum. Then we use the current total count rate to estimate the current background amplitude. From this amplitude we can correct the covariance matrix estimate from background tracking to current conditions of background amplitude, under the assumptions that spectrum channels are independent and observed channel counts are Poisson distributed. As a first step in the development, assume that the mean background spectrum at the current time step $k$ is a vector $\mathbf{u}_{\mathrm{k}}$ that is the product of a positive real scale factor $s_{k}$ and the estimated mean background from the EWMA tracking $\mathbf{u}_{\mathrm{k}}{ }^{\mathrm{o}}$. Under the above assumptions the likelihood of observing a measured spectrum $\mathbf{X}_{\mathrm{k}}$ is,

$$
P\left(\mathbf{X}_{k} \mid \mathbf{u}_{k}\right)={ }_{j=1}^{N} \frac{u_{k j}^{X_{k j}} e^{u_{k j}}}{X_{k j} !}
$$

where the product is over the $N$ channels in the spectrum. Maximizing this likelihood with respect to the scale factor $s$ gives,

$$
S_{k}=\frac{j^{X_{k j}}}{u_{k j}^{o}}=\frac{C_{k}}{B_{k}^{\text {tracking }}}
$$

where $C_{k}$ is the current observed total count rate and $B_{k}^{\text {tracking }}$ is the estimated total count rate extrapolated from the past to the present by EWMA. At locations where there is no systematic variability in background the covariance matrix is then approximately, 


$$
\begin{aligned}
& \boldsymbol{\Sigma}_{k}=\mathbf{T}_{k} \mathbf{A}\left(\begin{array}{cccccc}
u_{1} & 0 & . & . & . & 0 \\
0 & u_{2} & . & . & . & \cdot \\
\cdot & & . & & & \\
\cdot & & & . & & \\
\cdot & & & & . & \\
0 & 0 & . & . & . & u_{N}
\end{array}\right)\left(\mathbf{T}_{k} \mathbf{A}\right)^{T} \\
& \approx s_{k} \mathbf{T}_{k} \mathbf{A}\left(\begin{array}{cccccc}
u_{1}^{o} & 0 & \cdot & \cdot & . & 0 \\
0 & u_{2}^{o} & \cdot & \cdot & \cdot & \cdot \\
\cdot & & \cdot & & & \\
\cdot & & & \cdot & & \\
\cdot & & & & \cdot & \\
0 & 0 & . & . & \cdot & u_{N}^{o}
\end{array}\right)\left(\mathbf{T}_{k} \mathbf{A}\right)^{T} \approx s_{k} \Sigma_{k}^{\text {tracking }}
\end{aligned}
$$

where $\mathbf{T}_{\mathrm{k}}$ is the transformation from ROIs to SCRs reported in our earlier work (Pfund et al. 2010), and $\mathbf{A}$ is a matrix that combines counts in channels to give counts in ROIs. In (7) $\mathbf{T}_{k}$ is independent of the total count rate scale factor $s_{k}$. We have adopted the above approximation everywhere, with the understanding that it is asymptotically correct when the background changes in steps.

The correction to the covariance matrix changes each test statistic to the following,

$$
d_{i_{k}}^{2}=\boldsymbol{\alpha}_{i_{k}}^{\mathrm{T}} \cdot\left[r_{k}\left(\Sigma_{i_{k}}^{\text {tracking }}\right)^{-1}\right] \bullet\left(\mathbf{I}-\mathbf{P}_{i_{k}}\right) \bullet \boldsymbol{\alpha}_{i_{k}}
$$

where:

$$
r_{k}=\min \left\{\left(\frac{1}{s_{k}}\right), 1\right\} .
$$

With equation (9) we apply the correction $r_{k}$ conservatively -it acts only to reduce the test statistics and reduce alarm potential. 


\section{Replay and Injection Studies of Detection Algorithm Performance}

We assessed the impact of the covariance correction of N-SCRAD test statistics (8) - (9) by running the time series of San Diego spectra through the detection algorithm. The corrected variance estimates yielded distributions for N-SCRAD test statistics on the background data that were closer to ideal than what occurred when the correction was not used. As noted in an earlier publication (Pfund et al. 2010), excepting the fat tails, the test statistics should be, in theory, chi-distributed and location invariant. The chi-distribution for the alarm test statistic can be derived by approximating Poisson counts in ROIs as Gaussian, which is reasonable when those counts are greater than about 30. Linear transformations of the Gaussian counts vector produce standardized Gaussians, and the test statistic is the root sum of squares of those Gaussian random variables, producing a chidistributed variable. Plotted in the upper half of Figure 6 are histograms of N-SCRAD test statistics with five degrees of freedom, developed without applying the new correction factor, using test statistics (4) $\left(r_{k}=1\right)$. The dashed distribution was derived from a subsample of our data taken in urban San Diego. The dot-dashed distribution was derived from a subsample taken in La Jolla. The dotted line was derived from data from Wildcat Canyon. For comparison, the solid line is the chi-distribution for five degrees of freedom. The distributions from uncorrected N-SCRAD were skewed relative the chi-distribution - empirical alarm thresholds developed from them will be higher than expected for a given significance level. Plotted in the lower half of the figure are similar distributions obtained with the new correction factor applied according to (8). These distributions of test statistics were closer to theoretical chi-distribution, less skewed and more location-invariant. With the correction applied, alarm thresholds will be lower than without the correction.

The large test statistics for background spectra that occur when the correction is not used can be very localized and can have an operational impact when searching in such neighborhoods. The new covariance estimates can eliminate such distracting indications. Plotted in Figure 7 are test statistics resulting from search data collected by a person on foot. The data are not part of the San Diego data set. We present them here as an illustration of very large changes in background level - they were not used in our quantitative analysis. In this search the operator approached a structure of granite blocks several times - blocks that were significantly more 
radioactive in terms of total count rate than the surrounding land. The grey line are the test statistics from (4) plotted versus time that result when the new correction was not applied $\left(r_{k}=1\right)$. There were large anomaly indications in the neighborhood of the blocks. The black line gives the corresponding test statistics from (8) obtained with the correction applied. With the correction, there was no significant response from the granite blocks.

The lower alarm thresholds obtained with the covariance correction applied can result in higher detection rates for targets, depending on the allowed false positive rate. We injected spectral counts from ${ }^{137} \mathrm{Cs}$ sources and HEU sources at 409 random locations into the measured San Diego background data. The cesium targets differed in shielding from the cesium sources in the optimization set of 28. The HEU targets matched one of the 28 the ROIs were optimized for. Standoff distances at closest approach between the vehicle and each injection location were fixed at 3, 15 or 30 meters. At each standoff distance, 120 counts per second of the ${ }^{137}$ Cs target were delivered to the detector at closest approach. At each standoff distance, 150 counts per second of the HEU target were delivered to the detector at closest approach. To speed computations, roughly 31 targets were injected into each replay of the San Diego data, requiring roughly thirteen replays of the data for each set of 409 injections. Injections were constrained to be greater than 1000 meters or 1000 seconds apart from each other, and no injects were placed along freeways or Interstate highways. The vehicle was not assumed to move past targets in a straight line at constant speed - relative source-detector distances were calculated at each time step from the measured GPS coordinates in the San Diego data. In this comparative study, injected threat spectra were scaled with the square of vehicle to target distance, with no adjustments for attenuation or downscatter. The injected datasets were analyzed with N-SCRAD operating with either the covariance correction applied, or not. A true positive detection was counted for an injected target if one or more of the test statistics was above alarm thresholds, and the distance from the detection point to the target determined from the GPS coordinates was 35 meters or less, and only on the first pass of the vehicle by the target. Listed in columns 2 through 4 in Table 2 are alarm thresholds on three test statistics obtained when the correction was applied. Thresholds are shown for average nuisance and false positive rates (NFAR) of 1 per hour in the first two rows, roughly 1 per 2 hours in the 
second two rows, and roughly 1 per 4 hours in the last set of two rows, as estimated from the entire metro San Diego sample. Listed in parentheses are the estimated threshold values obtained when the new correction was not applied $\left(r_{k}=1\right)$. The thresholds needed to reach desired false positive rates with the correction were significantly lower than those estimated without it. Listed in columns 5 - 7 of the table are corresponding detection rates for 409 injections of ${ }^{137} \mathrm{Cs}$, at standoff distances of 3,15 and 30m. At an allowable NFAR of 1 per hour, we observed that probabilities of detecting ${ }^{137} \mathrm{Cs}$ increased significantly for source standoffs of 15 and $30 \mathrm{~m}$ when the correction was applied. Estimated probabilities for observing the results from the corrected algorithm with the uncorrected algorithm, assuming random, binomial trials were only $2 \%$ and $0.5 \%$ at 15 and $30 \mathrm{~m}$, respectively. At lower NFAR we also observed increased probabilities of detecting ${ }^{137} \mathrm{Cs}$ for the corrected algorithm at standoffs of 15 and 30m. The probabilities of chance observance of these improvements at an NFAR of 1 per 4 hours were only $0.4 \%$ and $0.06 \%$ (under the null hypothesis that detection is a Bernoulli process with the same success rate for both the improved and un-improved algorithms). The advantage of the corrected algorithm was more apparent as the allowed false positive rate was reduced. We note that in Table 2, probabilities of detection increased with distance - at a fixed maximum count rate at the detector at closest approach to the sources - because the exposure time to the detector increased with distance. Listed in columns $8-10$ of the table are the detection rates for the injections of HEU. For that source, the improvements in detection rates when the correction factor was applied were much smaller, but still positive.

It is interesting to compare target detection rates estimated by injection study for the optimized algorithm to rates obtained using the naïve square-root spaced ROIs and single alarm test statistic discussed in Section 4 . We configured the un-optimized detection algorithm to ignore signatures of K, $\mathrm{U}$, and Th-series nuisance sources and applied the covariance correction before calculating test statistics, as was done with the optimized algorithm. At an allowed NFAR of 1 per hour and a source standoff of 15-meters, the naïve version of the algorithm detected 45.7 percent of the injected ${ }^{137} \mathrm{Cs}$ targets and 55.0 percent of the injected HEU targets. These figures can be compared to the corresponding detection rates of 58.2 percent for ${ }^{137} \mathrm{Cs}$ and 69.9 percent for HEU from the 
algorithm using optimized ROI and test statistics. For both sources, detection rates increased by 27 percent as a result of parameter optimization, over what was obtained with the naïve parameters.

\section{Conclusions}

In this work we presented a process for improving the N-SCRAD algorithm in response to new applications or better awareness of operations. A component of the process was an improved optimization procedure for configuring the detection algorithm. The procedure was designed to obtain good overall sensitivity to a broad range of radiation signatures indicative of threats. The optimization by simulated annealing minimizes the size of the parameter set by discarding ineffective parameters, consistent with maintaining sensitivity to targets. It uses a large sample of measured background data as input, together with many simulated threat signatures selected to be relevant to real searches. The SA program is fast, enabling future development of multiple userselectable parameter sets for use in N-SCRAD under different detection scenarios.

We have also improved the detection algorithm to lower its response to unexpected changes in background. The improved algorithm gives higher detection rates for targets than does the original version, in cases when there is a low tolerance for nuisance and false positive alarms and the expected standoff distance is high.

\section{Acknowledgement}

Pacific Northwest National Laboratory is operated for the U.S. Department of Energy by Battelle under Contract DE-AC05-76RL01830.

This work is being supported by the U.S. Defense Threat Reduction Agency, under Interagency Agreement DTRA10027-10507. This support does not constitute an expressed or implied endorsement on the part of the Government. DISTRIBUTION A: Approved for public release: distribution unlimited (Ref. DTRA\# PA-15589/NT-15-786). 


\section{References}

Aage, H.K., Korsbech, U., Bargholz, K., Hovgaard, J., 1999. A new technique for processing airborne gamma ray spectroscopy for mapping low level contaminations. Appl. Radiat. Isot., 51, 651-662.

Aage, H.K., Korsbech, U., 2003. Search for lost or orphan radioactive sources based on NaI gamma spectroscopy. Appl. Radiat. Isot., 58, 103-113.

Anagnostopoulos, A., Michel, L., Van Hentenryck, P., Vergados, Y., 2006. A simulated annealing approach to the traveling tournament problem. J. Scheduling 9(2), 177-193.

Anderson, K.K, Jarman, K.D., Mann, M.L., Pfund, D.M., Runkle, R.C., 2008. Discriminating nuclear threats from benign sources in gamma-ray spectra using a spectral comparison ratio method. J. Radioanal. Nucl. Chem. 276(3), 713-718.

Chan, K.S., Li, J.Z., Eichenger, W., Bai, E.W., 2014. A distribution-free test for anomalous gamma-ray spectra. Radiat. Meas. 63, 18-25.

Ely, J.H., Kouzes, R.T., Schweppe, J.E., Siciliano, E.R., Strachan, D.M., Weier, D.R., 2006. The use of energy windowing to discriminate SNM from NORM in radiation portal monitors. Nucl. Instrum. Methods Phys. Res. A A560, 373-387.

Fagan, D.K, Robinson, S.M., Runkle, R.C., 2012. Statistical methods applied to gamma-ray spectroscopy algorithms in nuclear security missions. Appl. Radiat. Isot., 70, 2428-2439.

Guillot, L. 2001. Extraction of full absorption peaks in airborne gamma-spectrometry by filtering techniques coupled with a study of the derivatives. Comparison with the window method. J. Envir. Radioact. 53, 381-398.

Henderson, S.D., S. H. Jacobson, S.H., Johnson, A.W., 2003. The theory and practice of simulated annealing. In Handbook on Metaheuristics, International Series in Operations Research and Management Science 57, 287-319. 
Hendricks, T.J. 2001. An aerial radiological survey of abandoned uranium mines in Navaho Nation. Bechtel Nevada Remote Sensing Laboratory, Las Vegas, NV, Tech. Rep. DOE/NV/11718-602.

Hjerpe, T., Samuelsson, C., 2006. Shielded and unshielded geometries in the search for orphan sources. Appl. Radiat. Isot., 64, 551-555.

Jarman, K.D., Runkle, R.C., Anderson, K.K., Pfund, D.M., 2008. A comparison of simple algorithms for gammaray spectrometers in radioactive search applications. Appl. Radiat. Isot., 58, 103-113.

Jordan, D.V, Baciak, J.E., McDonald, B.S., Hensley, W.K., Miller, E.A., Wittman, R.S., Siciliano, E.R., 2011. Computational assessment of the impact of gamma-ray detector material properties on spectroscopic performance. Proc. SPIE 8142, Hard X-Ray, Gamma-Ray, and Neutron Detector Physics XIII, 81421K; doi:10.1117/12.897316

Kirkpatrick, S., Gelatt, C.D., Vecchi, M.P., 1983. Optimization by simulated annealing. Science 220(4598), 671680. Appl. Radiat. Isot., 68, 1832-1838.

Kock, P., Finck, R.R., Nilsson, J.M.C, Ostlund, K., Samuelsson, 2010. A deviation method for visualizing data in mobile gamma-ray spectrometry.

Los Alamos National Laboratory. PeakEasy Home Web Page [Online]. Available: http://peakeasy.lanl.gov.

MCNP X-5 Monte Carlo Analysis Team, "MCNP - A general purpose Monte Carlo n-particle transport code, version 5," Los Alamos National Laboratory LA-UR-03-1987, 2003.

Nelson, K.E., Gosnell, T.B., Knapp, D.A., 2011. The effect of energy resolution on the extraction of information content from gamma-ray spectra. Nucl. Instrum. Methods Phys. Res. A A659, 207-214.

Pfund D.M., Runkle, R.C., Anderson, K.K., Jarman, K.D, 2007. Examination of count-starved gamma spectra using the method of spectral comparison ratios. IEEE Trans. Nucl. Sci. 54(4), 1232-1238. 
Pfund, D.M., Jarman, K.D., Milbrath, B.D., Kiff, S.D., Sidor D.E., 2010. Low count anomaly detection at large standoff distances. IEEE Trans. Nucl. Sci. 57(1), 309-316.

Portnoy, D.A., Feuerbach, R.J., Heimberg, J.A., 2011. Global parameter optimization for maximizing radioisotope detection probabilities at fixed false alarm rates. Nucl. Instrum. Methods Phys. Res. A A652, $29-32$.

Press, W.H., Teukolsky, S.A., Vetterling, W.T., Flannery, B.P. 2002. Numerical Recipes in C++, 2nd ed. Cambridge, U.K.: Cambridge Univ. Press, 412-420.

Reinhart, A., Athey A., Biegalski, S., 2014. Spatially-Aware Temporal Anomaly Mapping of Gamma Spectra. IEEE Trans. Nucl. Sci. 61(3), 1284-1289.

Runkle, R.C., Smith, L.E., Peurrung, A.J., 2009a. The photon haystack and emerging radiation detection technology. J. Appl. Phys. 106, DOI: 10.1063/1.3207769.

Runkle, R.C., Myjak, M.J., Kiff, S.D., Sidor, D.E., Morris, S.J., Rohrer, J.S., Jarman, K.D., Pfund, D.M., Todd, L.C., Bowler, R.S., Mullen, C.A., 2009b. LYNX: An unattended sensor system for detection of gamma-ray and neutron emissions from special nuclear materials. Nucl. Instrum. Methods Phys. Res. A 598, 815-825.

Runkle, R.C., Tardiff, M.F., Anderson, K.K., Carlson, D.K., Smith, L.E., 2009c. Analysis of Spectroscopic Radiation Portal Monitor Data Using Principal Components Analysis. IEEE Trans. Nucl. Sci. 53(3), 1418-1423.

Suman, B., Kumar, P., 2006. A survey of simulated annealing as a tool for single and multiobjective optimization. J. Operational Res. Soc. 57, 1143-1160. 


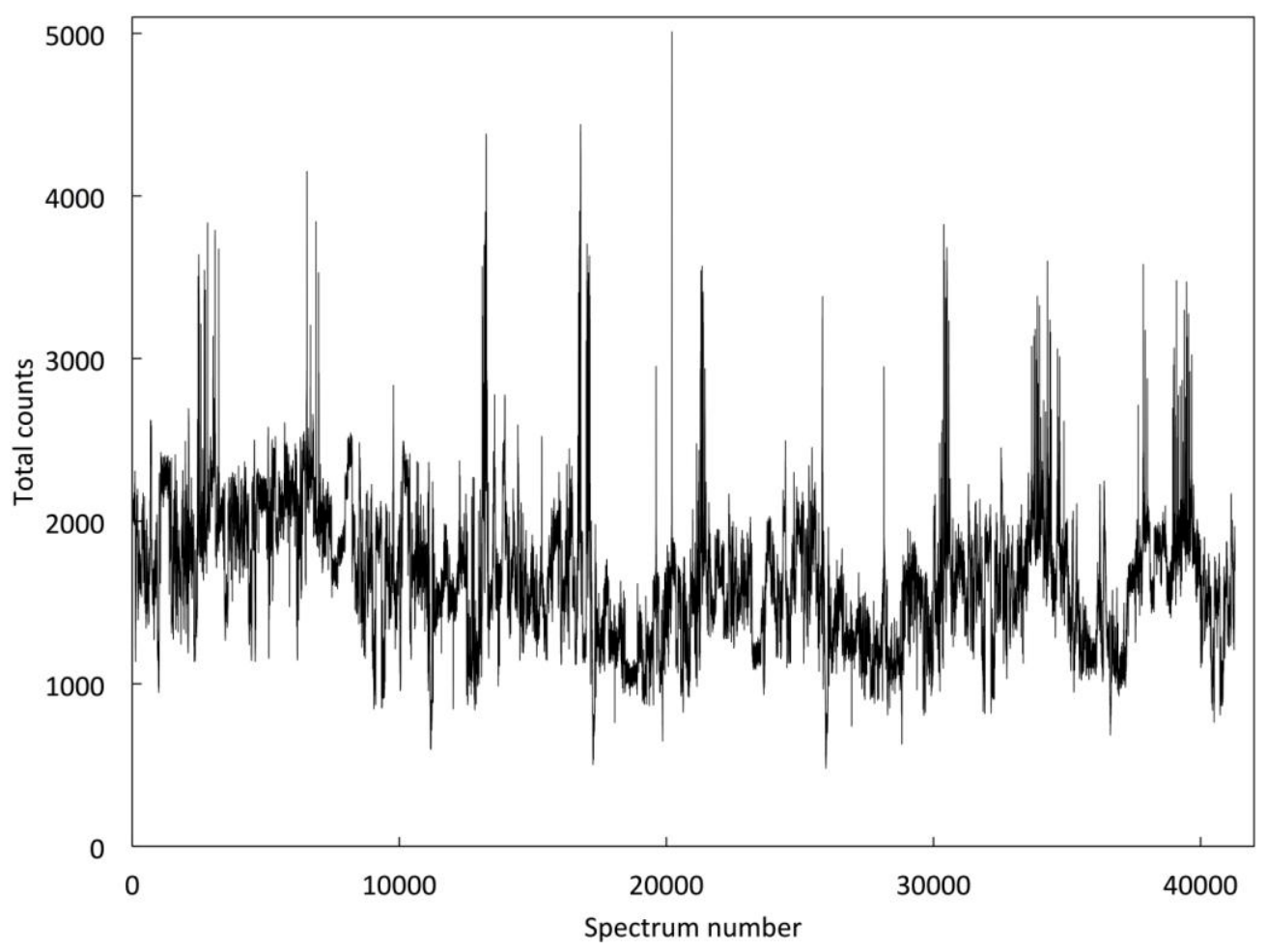

Fig. 1. Background total counts in the San Diego background data 

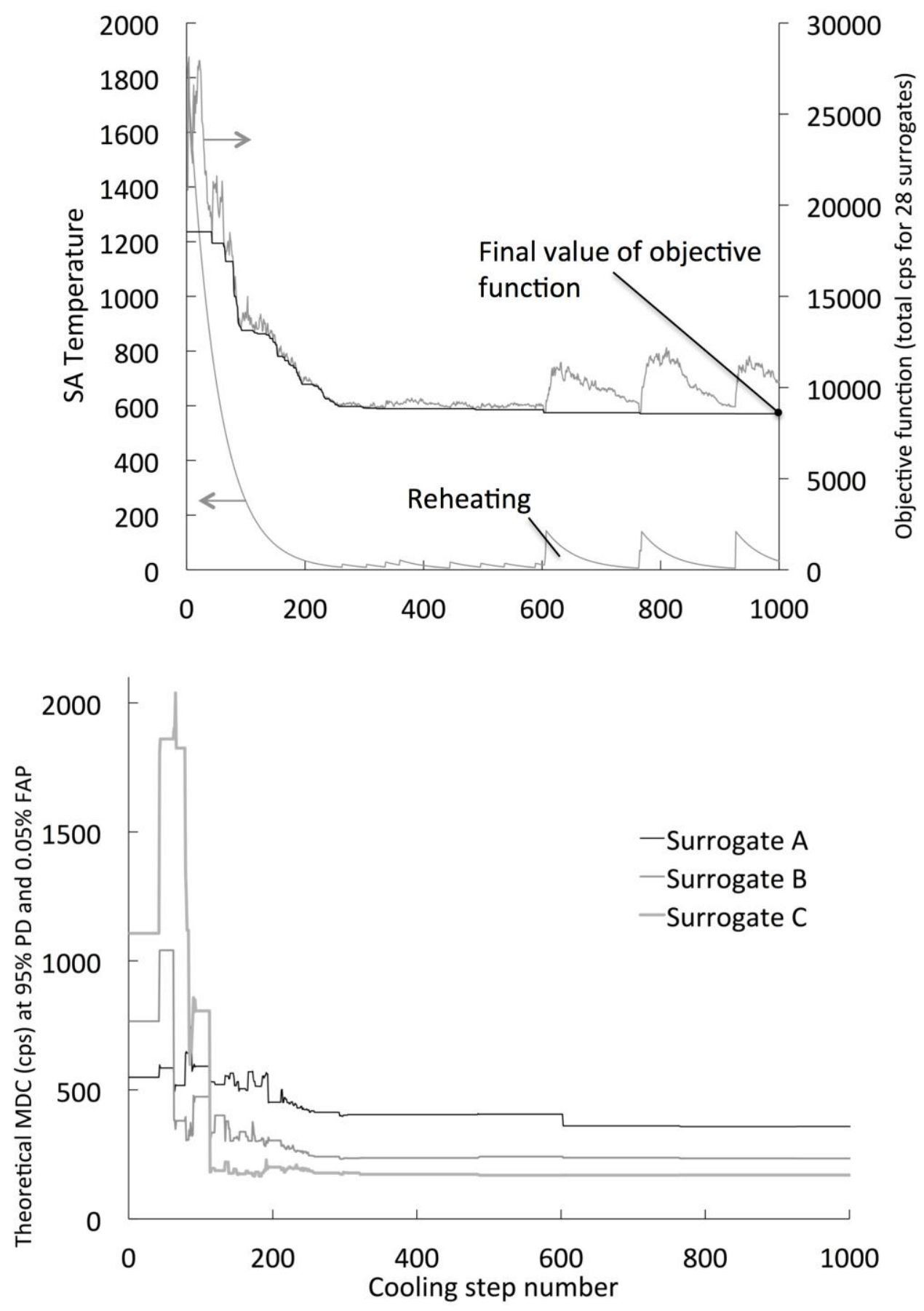

Fig. 2. Convergence behavior of the parameter optimization by simulated annealing. 

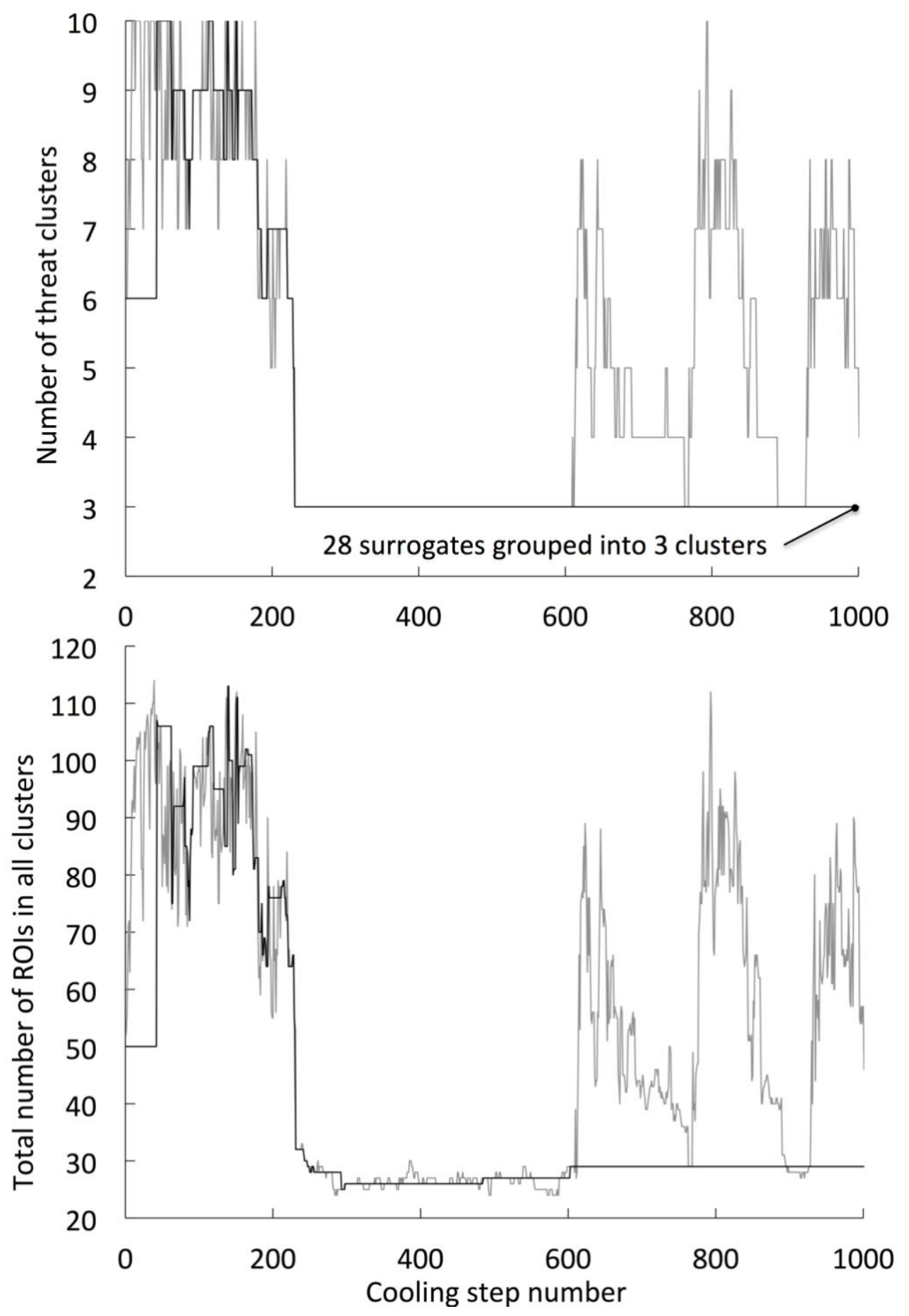

Fig. 3. Parameter reduction during the optimization. 


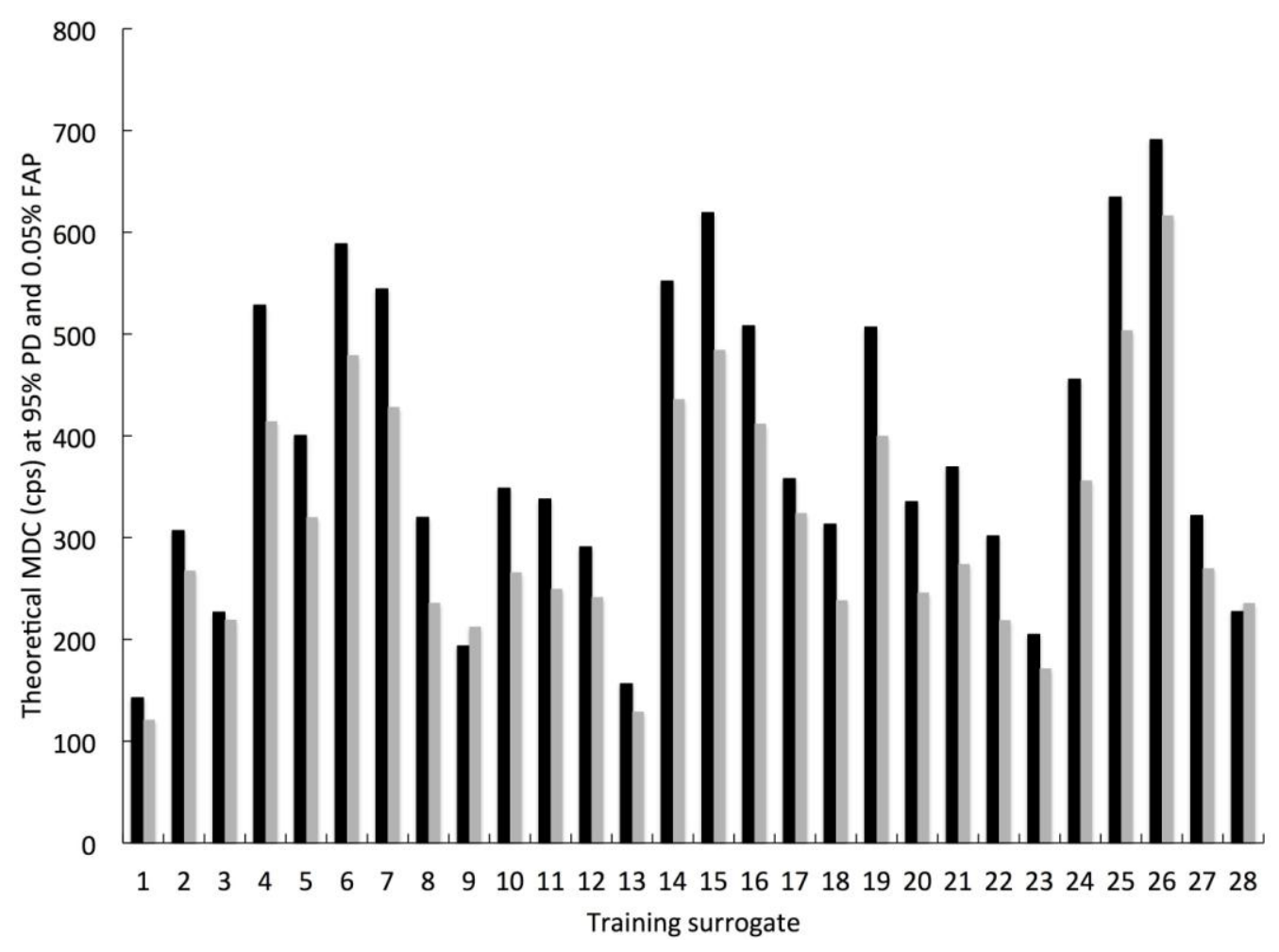

Fig. 4. Minimum detectable count rates for the 28 threat surrogates obtained using simple square-root spaced ROIs (black), compared to those obtained using optimized ROIs and test statistics (grey). 


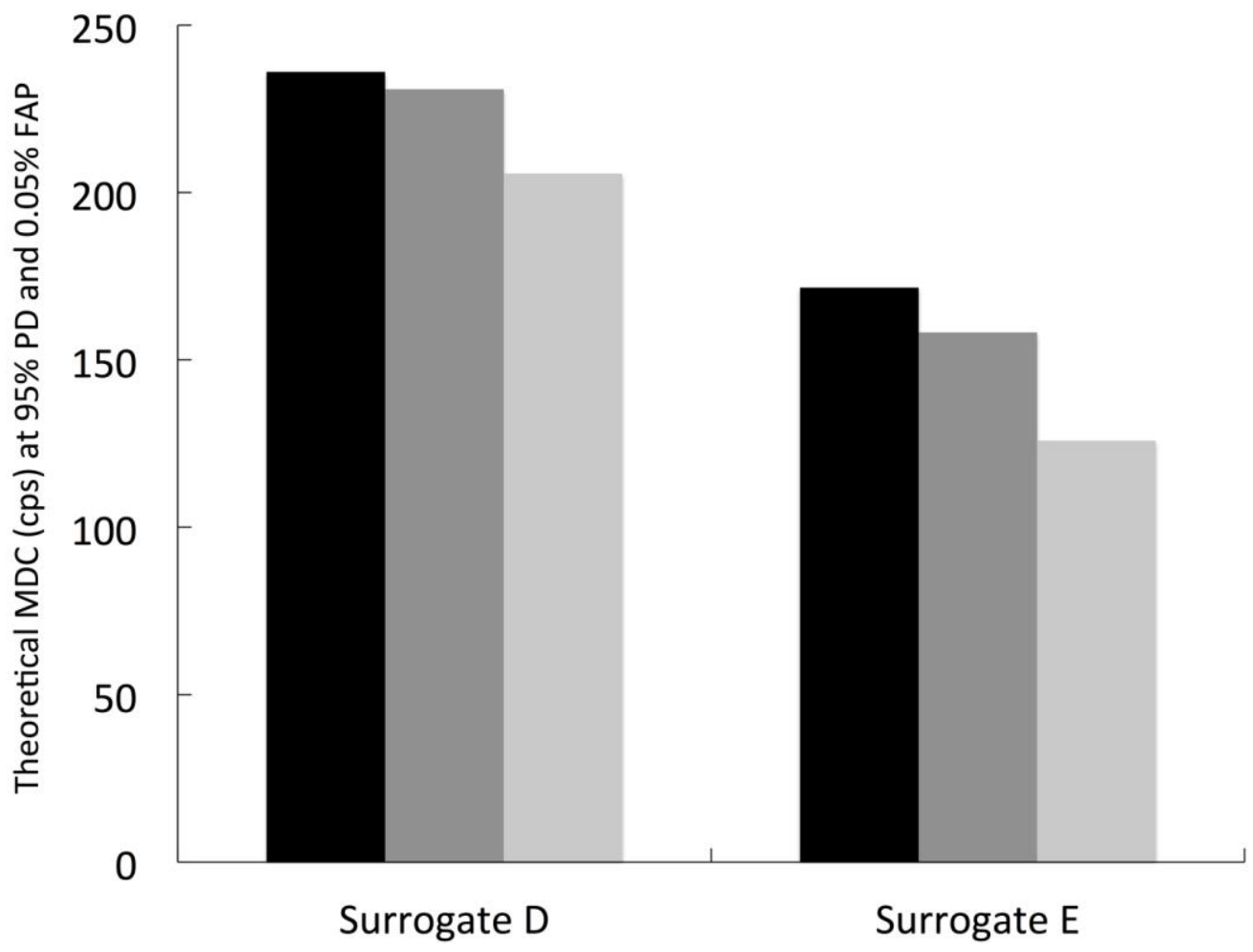

Fig. 5. Minimum detectable count rates for two threat surrogates under cases of broad (black), more narrow (dark grey), and single-source (light grey) parameter optimization. 


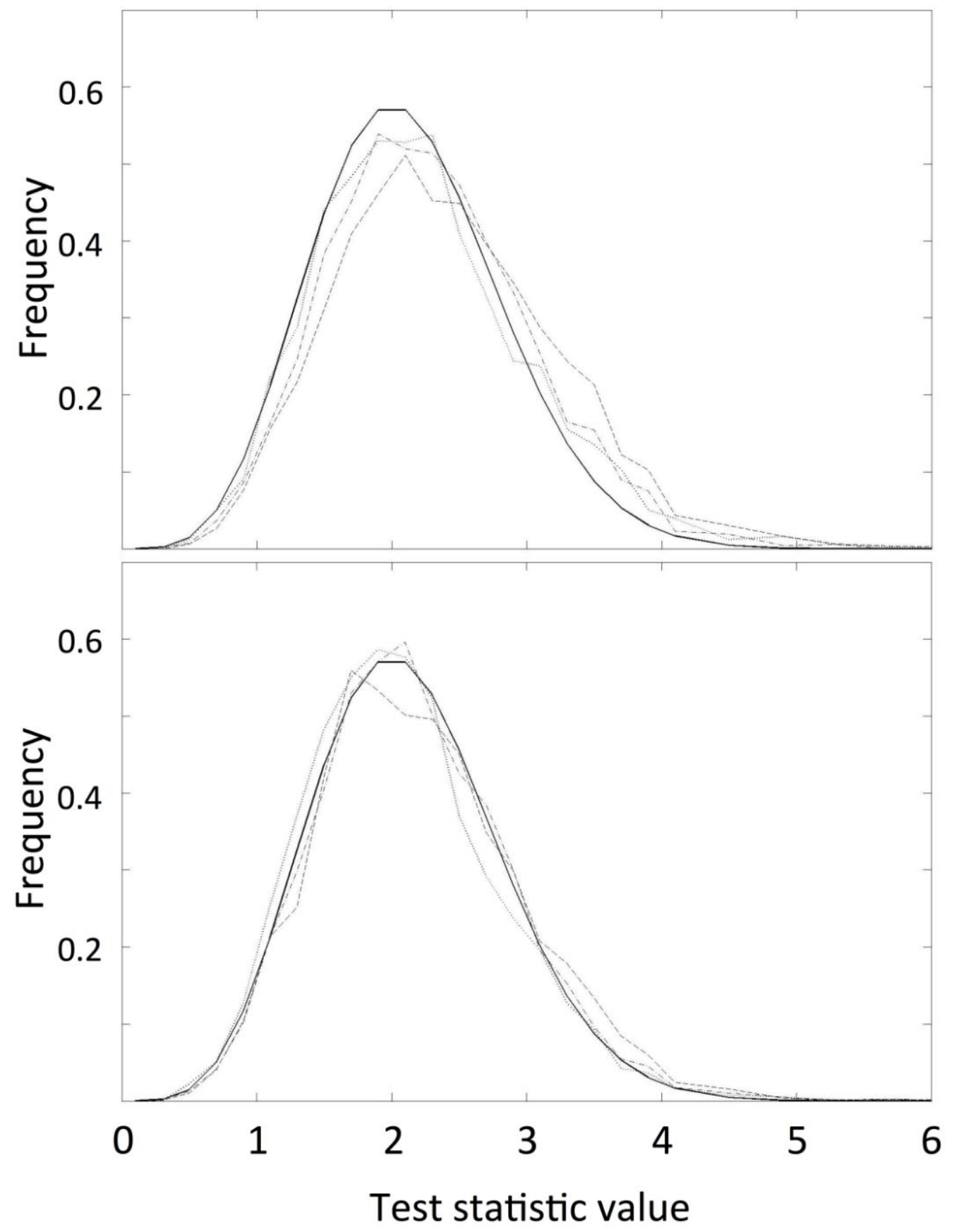

Fig. 6. Distributions of N-SCRAD test statistics in various geographic sub-populations compared to theory, with (bottom) and without (top) the new correction to the covariance matrix. 


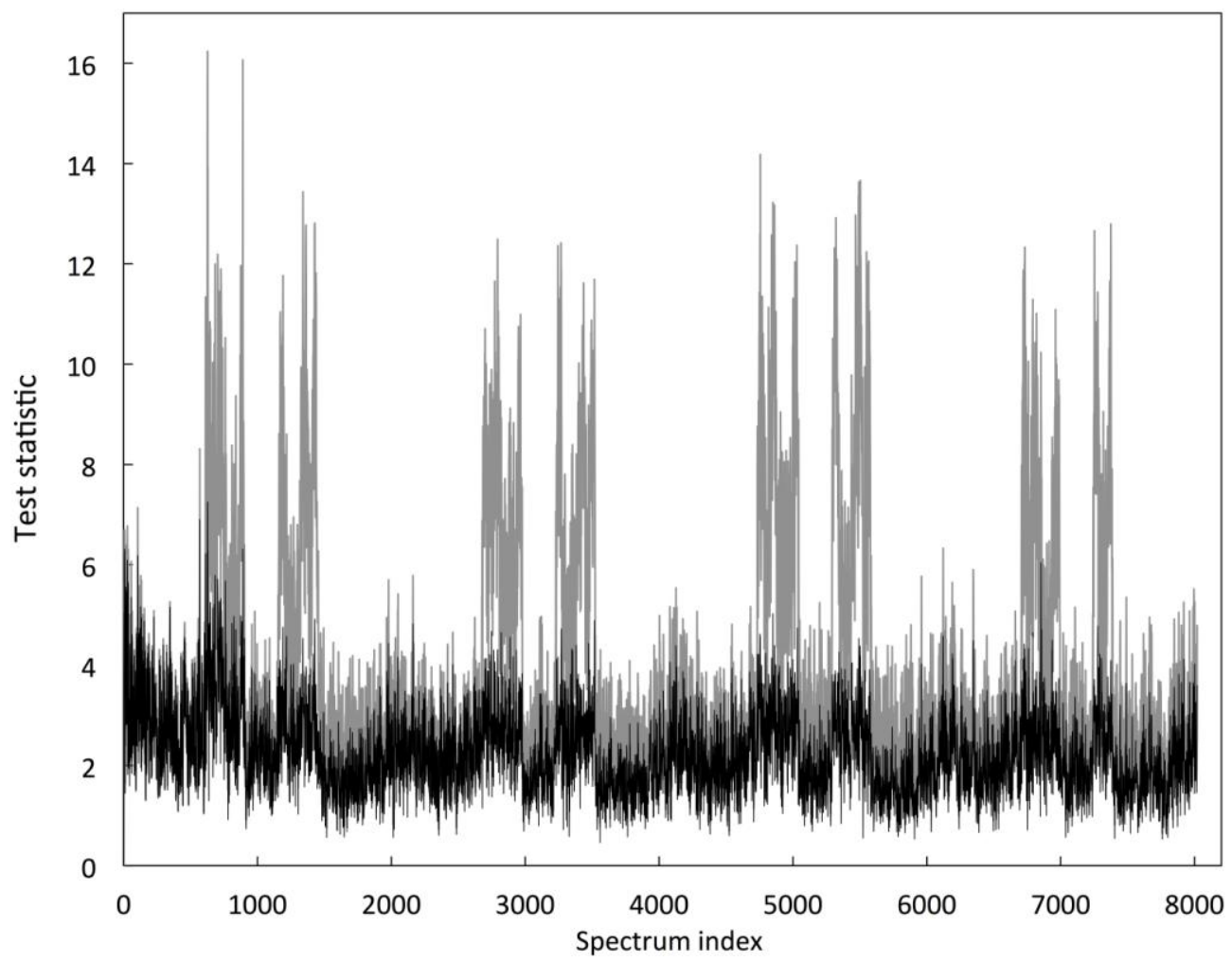

Fig. 7. Test statistic values for encounters with granite blocks, with (black) and without (grey) the new correction to the covariance matrix. 
Table 1. Parameters for simulated annealing.

Simulated Annealing Parameter

Value

Maximum number of active threat/ROI clusters

Maximum number of ROIs in a cluster

Required number of accepted moves before lowering the temperature

Ratio of new temperature to old temperature at each lowering 
Table 2. Detection performance with and without covariance correction.

\begin{tabular}{|c|c|c|c|c|c|c|c|c|c|}
\hline \multirow[b]{2}{*}{ NFAR } & \multicolumn{3}{|c|}{ Alarm Thresholds } & \multicolumn{3}{|c|}{${ }^{137}$ Cs Detection Percent } & \multicolumn{3}{|c|}{ HEU Detection Percent } \\
\hline & Metric 1 & Metric 2 & Metric 3 & $3 m$ & $15 \mathrm{~m}$ & $30 \mathrm{~m}$ & $3 \mathrm{~m}$ & $15 \mathrm{~m}$ & $30 \mathrm{~m}$ \\
\hline \multicolumn{10}{|l|}{1 per hour: } \\
\hline $\begin{array}{r}\text { Corrected } \\
\text { (Uncorrected) }\end{array}$ & $\begin{array}{c}6.168 \\
(6.998)\end{array}$ & $\begin{array}{c}5.714 \\
(7.095)\end{array}$ & $\begin{array}{c}5.079 \\
(5.699)\end{array}$ & $\begin{array}{c}27.4 \\
(23.7)\end{array}$ & $\begin{array}{c}58.2 \\
(51.1)\end{array}$ & $\begin{array}{c}73.9 \\
(65.6)\end{array}$ & $\begin{array}{c}36.2 \\
(32.3)\end{array}$ & $\begin{array}{c}69.9 \\
(67.0)\end{array}$ & $\begin{array}{c}81.5 \\
(81.0)\end{array}$ \\
\hline \multicolumn{10}{|l|}{0.6 per hour: } \\
\hline $\begin{array}{r}\text { Corrected } \\
\text { (Uncorrected) }\end{array}$ & $\begin{array}{c}6.207 \\
(7.621)\end{array}$ & $\begin{array}{c}6.196 \\
(7.209)\end{array}$ & $\begin{array}{c}5.120 \\
(5.772)\end{array}$ & $\begin{array}{c}26.2 \\
(21.3)\end{array}$ & $\begin{array}{c}53.3 \\
(45.5)\end{array}$ & $\begin{array}{c}66.3 \\
(56.6)\end{array}$ & $\begin{array}{c}35.5 \\
(30.6)\end{array}$ & $\begin{array}{c}68.9 \\
(65.5)\end{array}$ & $\begin{array}{c}80.7 \\
(80.2)\end{array}$ \\
\hline \multicolumn{10}{|l|}{0.3 per hour: } \\
\hline $\begin{array}{r}\text { Corrected } \\
(\text { Uncorrected })\end{array}$ & $\begin{array}{c}6.459 \\
(7.830)\end{array}$ & $\begin{array}{c}9.888 \\
(10.643)\end{array}$ & $\begin{array}{c}8.351 \\
(9.056)\end{array}$ & $\begin{array}{c}21.7 \\
(17.8)\end{array}$ & $\begin{array}{c}42.3 \\
(33.3)\end{array}$ & $\begin{array}{c}58.3 \\
(46.8)\end{array}$ & $\begin{array}{c}15.4 \\
(12.7)\end{array}$ & $\begin{array}{c}31.3 \\
(25.7)\end{array}$ & $\begin{array}{c}37.1 \\
(32.2)\end{array}$ \\
\hline
\end{tabular}

